\title{
Regadenoson SPECT MPI post-troponin elevation in two different patient populations: A reliable risk-stratification tool
}

\author{
Elona Rrapo Kaso, $M D,{ }^{a}$ and Jamieson $M$. Bourque, $M D, M H S^{a, b, c}$ \\ a Department of Medicine, University of Virginia Health System, Charlottesville, VA \\ b Department of Radiology, University of Virginia Health System, Charlottesville, VA \\ c Departments of Medicine and Radiology, Cardiovascular Imaging Center, University of Virginia \\ Health System, Charlottesville, VA
}

Received Apr 3, 2019; accepted Apr 3, 2019

doi: $10.1007 / \mathrm{s} 12350-019-01726-8$

\section{See related article, pp. 2320-2331}

Elevations in cardiac troponins identify multiple high-risk subgroups of patients including those who present with acute myocardial infarction (MI) without ST-segment elevation and those with unclear etiology for troponin rise. Both of these patient subgroups have an increased risk of mortality. In an 895-patient subset in the invasive arm from the Treat Angina With Aggrastat and Determine Cost of Therapy With Invasive or Conservative Strategy-Thrombolysis In Myocardial Infarction (TACTICS-TIMI)-18 trial, patients with positive troponin and $\geq 50 \%$ stenosis had the highest rate of 6-month mortality or re-infarction (8.6\%) vs $3.1 \%$ with a positive troponin but no obstruction and $0 \%$ with negative troponin. ${ }^{1}$ In addition, after an acute MI, the risk of heart failure, cardiac death, and re-hospitalization increases. A similar adverse prognosis is appreciated in those with elevated cardiac troponin of unclear etiology, a population whose prevalence has increased in the era of high-sensitivity cardiac troponin. Lee et al found that 1 in 8 patients without suspected ACS seen in the emergency department who received troponin testing had an elevated concentration. They confirmed prior research showing an association of

Reprint requests: Jamieson M. Bourque, MD, MHS, Departments of Medicine and Radiology, Cardiovascular Imaging Center, University of Virginia Health System, Box 800662, 1215 Lee Street, Charlottesville, VA, 22908; jamieson2@gmail.com

J Nucl Cardiol 202027:2332-6.

$1071-3581 / \$ 34.00$

Copyright (c) 2019 American Society of Nuclear Cardiology. troponin rise with mortality independent of age, sex, and renal function. ${ }^{2}$

Beyond troponin elevation, cardiac imaging is valuable in further risk stratification of these two patient populations. Assessment of myocardial perfusion and function and quantification of ischemia predict longterm outcomes and prognosis and separate low- and high-risk subsets with respect to future cardiac events. ${ }^{3}$ Nuclear single-photon emission computed tomography myocardial perfusion imaging (SPECT MPI) has a proven track record for diagnostic and prognostic value, with a large body of evidence of more than three decades. Left ventricular (LV) dysfunction, volume of infarcted and viable myocardium, and residual ischemia can be assessed accurately by stress SPECT MPI. SPECT MPI permits gating of images with calculation of left ventricular ejection fraction (LVEF), end-systolic and end-diastolic volumes and quantitation of regional wall motion or thickening abnormality extent. The percentage of LV ischemia, total perfusion deficit, and semi-quantitative grading of perfusion abnormalities using summed stress and difference scores separate patient subgroups by risk. ${ }^{3}$ SPECT MPI allows for timely in-hospital assessment of prognosis in the population with elevated troponin. It allows early hospital discharge for low-risk patients and more aggressive treatment, including invasive coronary angiography with possible coronary revascularization, for patients classified as high-risk by imaging parameters (see Figure 1).

With regard to mode of stress, pharmacologic SPECT MPI in particular has an important role and is used frequently for risk stratification in patients with acute elevation of troponin. In the first 48 hours of troponin elevation, exercise stress is unsafe and pharmacologic stress testing is the standard protocol (safe 24 hours after peak troponin). However, even more 


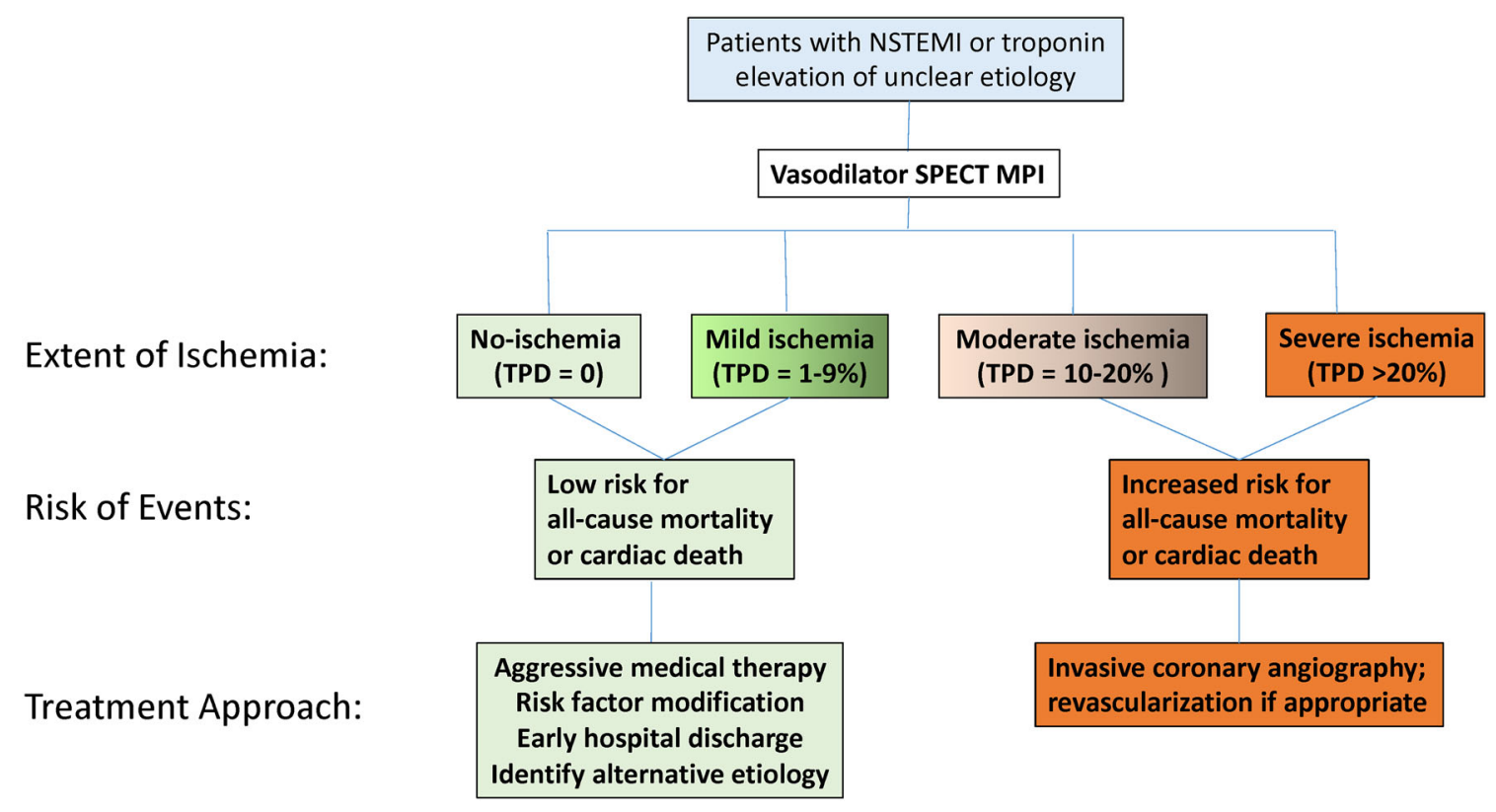

Figure 1. Risk stratification based on pharmacologic SPECT MPI in patients with positive troponin. NSTEMI, Non-ST elevation myocardial infarction; TPD, total perfusion defect.

than 48 hours after a troponin elevation, a significant portion of this patient population undergo pharmacologic stress testing either due to pre-existing resting ECG abnormalities such as left bundle branch block, poor mobility, contraindicated comorbidities, or due to an inability to achieve $85 \%$ of maximum age-predicted heart rate (MAPHR). Hashimoto et al showed that only $54 \%$ and $67 \%$ of patients older and younger than 75 years respectively were able to achieve $\geq 85 \%$ MAPHR. ${ }^{4}$ The prognostic information from vasodilator MPI in patients unable to adequately exercise has been shown to be similar to that with exercise stress MPI. ${ }^{5}$ There are currently three vasodilators approved for myocardial perfusion stress testing: dypiridamole, adenosine, and regadenoson. Dypiridamole and adenosine stress SPECT MPI both have an extensive body of literature with supportive evidence for a prognostic role after an acute MI. ${ }^{6,7}$ Currently, regadenoson is the most commonly-used vasodilator in the US, as it has fewer side effects and is easier to administer than the older vasodilators. ${ }^{8-10}$ Despite its frequent use in this population, the prognostic utility of regadenoson SPECT MPI in patients who have an elevated troponin after an acute non-ST elevation MI has not been established. Moreover, the prognostic role of regadenoson stress MPI in patients with elevated troponin of unclear etiology is not known.

In this issue of the Journal, Gowdar et $\mathrm{al}^{11}$ provide an important addition to the body of evidence validating SPECT MPI as a prognostic tool with incremental value to clinical and laboratory data. This retrospective cohort study in two tertiary medical centers evaluated the early use of regadenoson SPECT MPI for risk stratification in patients with elevated troponin after MI or in patients with troponin elevation of unclear etiology. This study is part of a series of investigations from this multicenter group who have previously shown that vasodilator stress testing in this cohort of patients is safe and feasible. Five hundred and three patients without prior coronary revascularization who had elevated troponin during index hospitalization and had MPI performed within 7 days were included. This current, well-designed study demonstrated that regadenoson SPECT MPI can be used reliably to risk stratify for cardiac mortality. The authors showed that increases in the extent and severity of perfusion defects on regadenoson stress MPI in patients with recently-elevated troponin are associated with higher event rates. Total perfusion defect (TPD) extent at stress was an independent predictor of both cardiac and all-cause mortality over a 33.6 month mean followup. Information on LVEF and TPD had a greater increase in prognostic value. TPD showed incremental prognostic value for all-cause mortality over cardiac risk factors, troponin level, and LVEF with a trend towards incremental value in cardiac mortality.

The results of the current study by Gowdar et al confirm and support the clinical use of regadenoson SPECT MPI for prognostic stratification which is essential beyond the diagnosis of coronary artery stenosis in the patient populations discussed above. As 
the COURAGE trial and FAME and DEFER studies have emphasized, ischemic presence and burden play an important role in management of coronary stenosis. ${ }^{12-14}$ For example, revascularization of non-significant lesions increases rates of cardiac events without improvements in angina. Indeed, similarly to prior robust data on adenosine SPECT MPI by Hachamovitch et al ${ }^{15}$ Gowdar et al show that the amount of perfusion abnormality at stress is a powerful predictor of cardiac death. Through quantification of ischemia and total perfusion defects, SPECT MPI identifies patients at low-, intermediateand high-risk for non-fatal MI and cardiac death. ${ }^{16}$ Moreover, SPECT MPI performs well in assessment of LVEF for further risk stratifications. A meta-analysis of nine studies with 164 subjects has shown that LVEF calculated by nuclear gated studies is highly-correlated with cardiac magnetic resonance (CMR) measurements; exceptions to this include women with smaller LV volumes and patients with dilated cardiomyopathy or global subendocardial perfusion defects. ${ }^{17}$ SPECT MPI, which is readily and widely available, safe, and feasible for risk stratification of troponin-positive patients, ${ }^{18}$ will continue to play a substantial role in providing reliable prognostic data in the era of high-sensitivity troponin.

It is also important to emphasize that, in contrast to the limitations of other anatomic and functional modalities, SPECT MPI is not limited by resting wall motion abnormality (WMA), ventricular pacing, atrial fibrillation, bundle branch blocks, or chronic kidney disease (CKD). Moreover, it is generally reproducible among different institutions and laboratories. Patients who have experienced an acute MI or who have positive troponin of unclear etiology have been shown to have elevated creatinine, which limits use of various imaging modalities such as CMR due to gadolinium contraindication and computed tomography (CT) due to nephrotoxicity from iodinated contrast. With regard to CKD, Lee et al showed that the group of patients with elevated troponin without ACS had a creatinine that was statistically higher compared to the group of patients with negative troponin. ${ }^{2}$ Large registries report that approximately $40 \%$ of patients with NSTEMI have CKD as defined by an estimated glomerular filtration rate (eGFR) $<60 \mathrm{~mL} / \mathrm{min}$ per $1.73 \mathrm{~m}$; of these patients, $22.6 \%$ have eGFR $<30 \mathrm{~mL} / \mathrm{min}$ per $1.73 \mathrm{~m} .{ }^{19}$ While CMR's greatest limitation is the use of gadolinium, which is contraindicated in patients with acute renal insufficiency or eGFR less than $30 \mathrm{~mL} / \mathrm{min} / 1.73 \mathrm{~m}^{2}$, CMR also has patient-specific limitations, including contraindications with most pacemakers/cardioverterdefibrillators, bore-fitting issues with substantial obesity, gating difficulties with irregular rhythms, inability to follow breath-hold instructions, and patient intolerance due to claustrophobia. Furthermore, CMR imaging expertise is not widely available. In those able to receive a successful scan and an expert interpretation, CMR allows for a comprehensive evaluation of myocardial structure, ventricular function and volume, myocardial perfusion, absolute myocardial blood flow, and detailed scar mapping, which also provides information on myocardial viability. CMR MPI is not limited by attenuation artifacts, yielding improved spatial resolution that better identifies myocardial scar compared with SPECT. It has been shown to provide excellent prognostic information in patients with known or suspected $\mathrm{CAD},{ }^{20}$ but the literature for prognostic value of CMR post-acute MI is less well developed when compared with stress SPECT MPI.

In addition to challenges with fitting in the bore of a MRI machine, obesity limits the quality of stress echocardiography significantly. This is unfortunate, as obesity has high prevalence in patients with acute MI. ${ }^{19,21}$ A 2012 analysis of the American Heart Association Get With The Guidelines Coronary Artery Disease program found that an obese body mass index (BMI) of $30 \mathrm{~kg} / \mathrm{m}^{2}$ or more is present in approximately $30 \%$ of patients admitted with NSTEMI. ${ }^{16}$ This rate of co-morbid NSTEMI will only increase as the prevalence of obesity rises. A more recent study in patients with suspected CAD showed that $47 \%$ of patients had a BMI $>30 \mathrm{~kg} / \mathrm{m}^{2} .18$

Stress echocardiography, although widely available, is limited by an accurate assessment of reduced regional systolic wall thickening induced by exercise or dobutamine especially in population with pre-existing WMA and/or in population with obesity due to poor sonographic windows. Furthermore, it is more challenging to quantify ischemia by stress echo when compared with SPECT MPI. When the prognostic value of pre-discharge dobutamine stress echocardiography (DSE) and dobutamine myocardial SPECT perfusion imaging were compared in 146 consecutive patients post a first acute uncomplicated MI, it was found that DSE ischemia was unable to stratify patients, whereas ischemia on SPECT MPI was associated with an increased risk of cardiac events during long-term follow-up. ${ }^{22}$ Other limitations of stress echocardiography include: ventricular pacing; bundle branch blocks; and operator-dependent technique. ${ }^{23}$

Current techniques in coronary computed tomographic angiography (CCTA) are primarily used for diagnosis of coronary artery stenosis but are greatly limited by the inability to directly assess the physiological significance of stenotic lesions. In fact, CCTA is rarely used for post-MI risk stratification because it does not assess ischemia. FFR $\mathrm{CT}_{\mathrm{T}}$ added to CCTA is a step towards overcoming the limitations of CCTA by allowing functional significance to be estimated noninvasively, utilizing CCTA anatomical imaging 
information and computational fluid dynamics to provide an estimated FFR. However, CT-FFR is limited as it is an indirect measurement of myocardial ischemia and does not assess for microvascular disease. Although FFR has incremental prognostic value when combined with CCTA, none of these methods provides information regarding myocardial perfusion, which, as discussed earlier, is crucial information for risk stratification and management of coronary artery stenosis. In addition, a recent study has shown that assessment of perfusion (in this case by CTA) has higher prognostic value than CCTA alone or with CT-FFR. ${ }^{24}$ Although dynamic myocardial perfusion can be assessed with new CTA techniques, its use is predominately limited to research without wide clinical application.

The strong predictive value of perfusion assessment is valuable not only for ischemia due to epicardial coronary artery stenosis, but also for identification of microcirculatory impairment. PET MPI has improved spatial resolution, higher count sensitivity, and more accurate soft issue attenuation correction compared to SPECT, allowing better imaging quality regardless of body habitus. ${ }^{25}$ PET has the advantage of providing prognostic information regarding myocardial blood flow in absolute units, which is important in the assessment of the distal coronary microcirculation. ${ }^{26}$ Its assessment of absolute flow and other advantages provide increased ability to identify left main and multivessel obstructive CAD. ${ }^{26}$ However, cardiac PET is not widely available due to the high cost of cameras and the high cost and limited availability of cyclotrons and rubidium generators. Accessibility will potentially improve in the future with new radiotracers such as ${ }^{18} \mathrm{~F}$-flurpiridaz, which could be distributed more widely and has shown high utility in a phase-3 study with a second study underway to qualify for FDA approval.

This current study by Gowdar et al has substantial strengths. It has a robust sample size and appropriate statistical analysis with adequate adjustment for baseline variables. Even after Cox proportional hazards analysis was performed with adjustment for multiple covariables including age, gender, BMI, and cardiac risk factors, the authors identified that increasing TPD percentage predicts both cardiac and all-cause mortality. There were many predictors of cardiac mortality and all-cause mortality with no interaction with TPD size on pharmacologic stress. This study shows that regadenoson SPECT MPI can be utilized reliably for risk stratification in patients with positive troponin. Another strength of this study is that the pharmacologic SPECT MPI was interpreted at the time of the patient's clinical presentation and outcome data were collected retrospectively, which reduces bias in the interpretation of SPECT MPI (since the interpreting cardiologists did not know at the time that their interpretations would be used for this particular research study). Also, the mean length of follow-up approached 3 years. Importantly, the results of this study are consistent with prior publications showing the prognostic utility of vasodilator SPECT MPI with adenosine and dipyridamole. ${ }^{6,7}$

As with any study, there are limitations and residual questions remain. The authors do not provide information on non-fatal MI or re-infarction rates. As previously shown in 1008 patients who underwent adenosine SPECT MPI and were followed over a mean of 2 years, the magnitude of perfusion defect reversibility remained the strongest predictor of non-fatal MI, whereas cardiac death was best predicted by post-stress LV ejection fraction, even after adjustment for clinical factors. ${ }^{27}$ Whether this relationship also holds true with regadenoson in this population of patients with elevated cardiac biomarkers is not answered by Gowdar et al and is a question for future study. In addition, as the authors appropriately point out, the cardiac mortality obtained from the National Death index has limitations that may reduce its accuracy. Moreover, it would be illuminating to separate the patients with elevated troponin post-MI from those with unexplained troponin elevations and compare the risk stratification data between these two groups. What are the characteristics of the population with elevated biomarkers of uncertain etiology? What is the prevalence in this sub-population? Is the prognostic value of MPI in patients who have elevated troponin after acute MI similar to patients with troponin of uncertain etiology? These important questions should be addressed in future studies.

This analysis adds to a growing body of highquality, high-impact evidence confirming the incremental prognostic value of vasodilator stress MPI after acute MI and for the first time shows the prognostic value of regadenoson stress MPI after uncomplicated acute MI and troponin elevation of unclear etiology. We look forward to additional research in this important patient population.

\section{Disclosure}

J. Bourque is a consultant for Pfizer and receives research grant support from Astellas. He has an ownership interest in Locus Health. E. Rrapo Kaso has nothing to disclose.

\section{References:}

1. Dokainish $\mathrm{H}$, et al. Prognostic implications of elevated troponin in patients with suspected acute coronary syndrome but no critical 
epicardial coronary disease: A TACTICS-TIMI-18 substudy. JACC 2005;2005:19-24.

2. Lee, et al. Prevalence, determinants, and clinical associations of high-sensitivity cardiac troponin in patients attending emergency departments. Am J Med 2019;132:110.e8-21.

3. Bourque JM, Beller GA. Stress myocardial perfusion imaging for assessing prognosis: An update. JACC Cardiovasc Imaging 2011;4:1305-19.

4. Hashimoto, et al. Complications of exercise and pharmacologic stress tests: Differences in younger and elderly patients. J Nucl Cardiol 1999;6:612-9.

5. Navare SM, Mather JF, Shaw LJ, Fowler MS, Heller GV. Comparison of risk stratification with pharmacologic and exercise stress myocardial perfusion imaging: a meta-analysis. J Nucl Cardiol 2004;11:551-61.

6. Mahmarian JJ, Shaw LJ, Filipchuk NG, Dakik HA, Iskander SS, Ruddy TD, et al. A multinational study to establish the value of early adenosine technetium-99m sestamibi myocardial perfusion imaging in identifying a low-risk group for early hospital discharge after acute myocardial infarction. J Am Coll Cardiol 2006;48:2448-57.

7. Brown KA, Heller GV, Landin RS, Shaw LJ, Beller GA, Pasquale MJ, et al. Early dipyridamole $(99 \mathrm{~m}) \mathrm{Tc}$-sestamibi single photon emission computed tomographic imaging 2 to 4 days after acute myocardial infarction predicts in-hospital and postdischarge cardiac events: comparison with submaximal exercise imaging. Circulation 1999;100:2060-6.

8. Iqbal FM, Hage FG, Ahmed A, Dean PJ, Raslan S, Heo J, et al. Comparison of the prognostic value of normal regadenoson with normal adenosine myocardial perfusion imaging with propensity score matching. JACC Cardiovasc Imaging 2012;5:1014-21.

9. Al Jaroudi W, Iskandrian AE. Regadenoson: A new myocardial stress agent. J Am Coll Cardiol 2009;54:1123-0.

10. Farzaneh-Far A, Shaw LK, Dunning A, Oldan JD, O'Connor CM, Borges-Neto $\mathrm{S}$. Comparison of the prognostic value of regadenoson and adenosine myocardial perfusion imaging. J Nucl Cardiol 2015;22(4):600-7.

11. Gowdar S, Ahlberg AW, Rai M, et al. Risk stratification with vasodilator stress SPECT myocardial perfusion imaging in patients with elevated cardiac biomarkers. J Nucl Cardiol 2019. https://doi. org/10.1007/s12350-019-01661-8.

12. Shaw LJ, Berman DS, Maron DJ, Mancini GBJ, Hayes SW, Hartigan PM, et al. Optimal medical therapy with or without percutaneous coronary intervention to reduce ischemic burden: results from the Clinical Outcomes Utilizing Revascularization and Aggressive Drug Evaluation (COURAGE) trial nuclear substudy. Circulation 2008;117:1283-91.

13. Pijls NHJ, Fearon WF, Tonino PAL, Siebert U, Ikeno F, Bornschein B, et al. Fractional flow reserve versus angiography for guiding percutaneous coronary intervention in patients with multivessel coronary artery disease: 2-year follow-up of the FAME (Fractional Flow Reserve Versus Angiography for Multivessel Evaluation) study. J Am Coll Cardiol 2010;56:177-84.

14. Pijls NHJ, van Schaardenburgh P, Manoharan G, Boersma E, Bech $\mathrm{J}-\mathrm{W}$, van't Veer M, et al. Percutaneous coronary intervention of functionally nonsignificant stenosis: 5-Year follow-up of the DEFER study. J Am Coll Cardiol 2007;49:2105-11.
15. Hachamovitch R, Berman DS, Shaw LJ, et al. Incremental prognostic value of myocardial perfusion single photon emission computed tomography for the prediction of cardiac death: Differential stratification for risk of cardiac death and myocardial infarction. Circulation 1998;97:535-43.

16. Shaw LJ, Iskandrian AE. Prognostic value of gated myocardial perfusion SPECT. J Nucl Cardiol 2004;11:171-85.

17. Ioannidis JPA, Trikalinos TA, Danias PG. Electrocardiogramgated single-photonemission computed tomography versus cardiacmagnetic resonance imaging for the assessment of left ventricular volumes and ejection fraction: A meta-analysis. J Am Coll Cardiol 2002;39:2059-68.

18. Rai M, Ahlberg AW, Marwell J, Chaudhary W, Savino JA 3rd, Alter EL, et al. Safety of vasodilator stress myocardial perfusion imaging in patients with elevated cardiac biomarkers. J Nucl Cardiol 2017;24:724-34.

19. Fox CS, Muntner P, Chen AY, et al. Use of evidence-based therapies in short term outcomes of ST-segment elevation myocardial infarction and no-ST-segment elevation myocardial infarction in patients with chronic kidney disease: a report from National Cardiovascular Data Acute Coronary Treatment and Intervention Outcomes Network registry. Circulation 2010;121:357-65.

20. Hamirani YS, Kramer CM. Cardiac MRI assessment of myocardial perfusion. Fut Cardiol 2014;10:349-58.

21. Ouellette ML, Löffler AI, Beller GA, Workman VK, Holland E, Bourque JM. Clinical, sex differences, and outcomes in patients with normal or near-normal coronary arteries, non-obstructive or obstructive coronary artery disease. J Am Heart Assoc 2018;2:7.

22. Acampa, et al. Prognostic value of myocardial ischemia in patients with uncomplicated acute myocardial infarction: direct comparison of stress echocardiography and myocardial perfusion imaging. J Nucl Med 2005;46:417-23.

23. Pellikka PA, Nagueh SF, Elhendy AA, et al. American Society Echocardiography recommendations for performance, interpretation and application of stress echocardiography. JASE 2007;20:1021-41.

24. van Assen M, De Cecco CN, Eid M, et al. Prognostic value of CT myocardial perfusion imaging and CT-derived fractional flow reserve for major adverse cardiac events in patients with coronary artery disease. J Cardiovasc Comput Tomogr 2019. https://doi.org/ 10.1016/j.jcct.2019.02.005

25. Di Carli MF, Hachamovitch R. New technology for noninvasive evaluation of coronary artery disease. Circulation 2007;115:146480.

26. Dorbala S, Di Carli MF, Beanlands RS, et al. Prognostic value of stress myocardial perfusion positron emission tomography: results from a multicenter observational registry. J Am Coll Cardiol 2013;61:176-84.

27. Sharir T, Germano G, Kang X, et al. Prediction of myocardial infarction versus cardiac death by gated myocardial perfusion SPECT: risk stratification by the amount of stress-induced ischemia and the poststress ejection fraction. J Nucl Med 2001;42:8317.

Publisher's Note Springer Nature remains neutral with regard to jurisdictional claims in published maps and institutional affiliations. 